RAIRO-Oper. Res. 55 (2021) 395-413

https://doi.org/10.1051/ro/2021002

RAIRO Operations Research

www.rairo-ro.org

\title{
A NEW TENSOR APPROACH OF COMPUTING PURE AND MIXED UNILATERAL SUPPORT EQUILIBRIA
}

\author{
Elias Safatly* AND JoAnna E. AbDOU
}

\begin{abstract}
In this paper, pure unilateral support equilibrium (USE) is located among pure Nash and pure Berge equilibrium using tensors. The differences between these equilibria are shown using tensor form of a game and are illustrated with numerical examples. Tensors will help specify the location of each equilibrium using a system of coordinates that brings a solid mathematical foundation of all equilibria and provides the possibility to solve high dimensional problems. A numerical example with a 15-player game is studied to demonstrate the efficiency. Besides, we extend the notion of pure USE to mixed USE when the sets of strategies of all players are finite. We prove a lemma dedicated to inaugurate a method of computing mixed USE profiles. We write corresponding formulas using tensors and their operations, and then we illustrate the new method and lemma method by a numerical example of a 7 -player game.
\end{abstract}

Mathematics Subject Classification. 91A99.

Received January 17, 2020. Accepted January 2, 2021.

\section{INTRODUCTION}

Game theory provides a model for scientifically addressing strategic decision-making issues that are common in business, and often very complex. It helps understand the logic of the situations in which the consequences of players' actions depend on a set of actions taken by others, each trying to influence or guess the behavior of others, or adapt to it. To give solutions for players interactions, game theory is based on various notions of equilibrium; everyone reflects the way of coordination between rational players and their actions on a state with certain stability. However, every notion of equilibrium is postulated overhang the game by a state of equilibrium being subjected to the necessary condition that, if the players are there, they perceive no interest to deviate from their current strategy choices. One of the most used equilibria in the applications of game theory to many scientific fields, in particular to economics, is the Nash equilibrium; see [24]. When the concept of Nash equilibrium started to appear, the economists, then, preferred the work of Von Neumann, see [27], who proposed a theory of formal games, showing that in zero-sum games with two players, there would always be an equilibrium. They, first, neglected the Nash proposed work. Nevertheless, since 1980, the number of papers citing the Nash equilibrium have dramatically increased, and the concept has been used to solve several realworld problems in many fields. Nash equilibrium is a set of strategies where no one has any interest in deviating

Keywords. Game theory, Pure unilateral support equilibrium, mixed unilateral support equilibrium, Berge equilibrium, Nash equilibrium, tensors.

Faculty of Economics and Business Administration, Lebanese University, Beirut, Lebanon.

*Corresponding author: elias.safatly@ul.edu.lb 
from his equilibrium strategy when he expects others to conform to it. Such as what is the best strategy for each depends on what is the best for others. In Nash equilibrium, the optimal strategy of each will be the best response to the strategies of others. The equilibrium will be reached if no player has an interest in unilaterally changing his decision. A Nash equilibrium is based on the idea that players are rational. Players choose the strategy that maximizes their payoffs, given all constraints.

Nash equilibrium is studied in a huge number of papers, and covered in many disciplines. In addition, it gives solutions for many social-economics problems. However, it shows some weak points. For example, consider a game that has more than one Nash equilibrium. A player $i$ will risk, when choosing a strategy relative to one of these equilibria, to lose his optimal payoff when other players choose a strategy relative to another Nash equilibrium. This choice can destruct the player $i$ 's optimal gain and preserve the optimal gains of other players. Another weak point is the selfish behavior of players that could arise when mutual support behavior is available. In all the history of Nash equilibrium problems, selfish behavior of players and their attempt to maximize their own payoffs against other players were the main factor in the gain that the concept of Nash equilibrium offers for players. Unfortunately, in some cases, selfish behavior can not lead to receiving higher payoffs. Mutual support and reciprocal altruism, see [32], are concepts found for an equilibrium having the role to be the complementary of Nash equilibrium in many games where Nash equilibrium shows his weak point. This equilibrium is called Berge equilibrium and is based on altruistic mutual support behavior, see [8]. French mathematician Claude Berge introduced this equilibrium in [5]. Similar to John Nash, economists rejected the use of this book's ideas at the beginning. In 1961, the economist Shubik, who pointed Berge out, confirmed it in his famous statement "the argument is presented in a highly abstract manner and no consideration is given to applications to economics". In 1985, the first reference to [5] finally appeared with the Russian mathematician Zhukovskii, who reformulated the Berge coalitional equilibrium and restricted coalitions to individualistic players, see [33]. Then, since 2004, Berge equilibrium has led to a revolution in its properties, existences, utilities, and advantages as studied in $[1,2,7-13,21,25,26]$ and many other papers.

Recently, a new equilibrium concept is proposed in [30] and in [14]. It is called unilateral support equilibrium. This new equilibrium generalizes the idea of Berge equilibrium, since every Berge equilibrium is also a unilateral support equilibrium as shown in [14,30]. In a game of $n$ players, a Berge equilibrium is based on the idea of group mutual support where a player $i$ is supported by the group of $n-1$ remaining players. However, in unilateral support equilibrium, every player $i$ is supported by every other player $j \neq i$ individually, for all $j=1, \ldots, n$. However, Berge equilibrium can be criticized for involving a non-realistic strong coordination between players, see $[15,30]$. The concept of unilateral support equilibrium retains supportive behavior, although coordination is not assumed. For this reason, generalizing the Berge equilibrium is interesting while eliminating coordination issues. In [14], a real life example on unilateral support equilibrium is given. It considers big charities' sectors to show the importance of this equilibrium where Berge equilibrium can not be a choice because of huge number of donators. The level of cooperation, in this case, can be unfeasible.

In this paper, we help locate pure unilateral support equilibrium with a mathematical object provided by the multi-linear algebra called tensor. Authors in [18] worked in this field and reformulated the multi-linear game as a tensor complementarity problem and showed the correspondence between finding a Nash equilibrium point of this multi-linear game and finding a solution to the resulted tensor complementarity problem. Introducing tensors will facilitate the procedure to find pure equilibrium and will give a formalized mathematical aspect for the game using its tensor form introduced for the first time [3]. In [30], the authors proposed Trimatrix games and used cubical representation for these games in order to highlight their unilateral support equilibrium. These cubical representations and Trimatrix games can be generalized mathematically using tensors. Tensors are multi-linear forms that give the solid mathematical foundations and generalize Trimatrix games and their representations. Also, as a consequence of tensor forms is the validation of the relation between USE and Berge equilibrium as raised in [30]. Using just this tensor procedure, we can clearly confirm that every Berge equilibrium is a unilateral support equilibrium. In addition, a big advantage of tensor form is the capability to deal with high dimensional problems, especially when the number of players is greater than 3, which has not been studied in USE literature. In this paper, the implementation of USE algorithm is presented based 
on tensors as main variables. We extend as well the notion of pure unilateral support equilibrium to mixed unilateral support equilibrium when the set of strategies of each player is discrete and finite in non-cooperative games. A method to calculate the mixed unilateral support equilibrium in this case is also shown, based on [28]. We prove a lemma demonstrating this method, where we show that for each player $i$, every strategy in the support of an equilibrium mixed strategy of each player $j \neq i$, individually, yields that player $i$ the same payoff. Moreover, we use tensors form and operations to facilitate the computation of mixed unilateral support equilibrium.

In Section 2, we write tensors definitions, useful properties and operations used to define tensor form of a game giving a mathematical formalized aspect to game theory by helping locate mathematically pure and mixed equilibrium as seen in $[3,29]$.

In Section 3, we recall pure Nash and pure Berge equilibria definitions before writing the pure unilateral support equilibrium definition using tensor form, in order to clarify the difference between these concepts. Numerical examples are solved to show tensor form's utility. Moreover, we show using the tensor form, that each Berge equilibrium, is a USE and the interpretations of all these types of equilibria on tensors can be visualized geometrically, presenting all these concepts together on a tensor node. All the mathematical relations and the deviations of these equilibria can be assessed and deduced much more easily than the handling of their formulas.

Efficiency of tensor form is demonstrated in Section 4 by solving a high dimensional problem displayed by a 15-player game where we implement an algorithm based on tensors as main variables to localize a pure unilateral support equilibrium when it exists.

Finally, Section 5 provides the extension of pure unilateral support to mixed unilateral support equilibrium when the sets of strategies of each player are discrete and finite. We define a new set called Best Individualistic Support, in order to inaugurate a method dedicated to calculate mixed unilateral support equilibrium profiles. This method is generated by a lemma that will be introduced and proved. Then, it will be implemented (using a tensor multiplication with vectors) and applied to a 7-player game.

\section{TEnsor FORM OF A GAME}

Tensors came from the world of multi-linear algebra and pure mathematics, see [6,31]. It is used in game theory in order to give mathematical organization to all game theory problems and deal with high dimensional mixed and pure equilibrium problems. In this section, we define the tensor form of a game initially introduced for the first time in [3] where we found that tensor form of a game gives a mathematical definition for all games $\Gamma_{n}=<I,\left(Z_{i}\right)_{i \in I},\left(p_{i}\right)_{i \in I}>, \forall n \geq 3$, where the set of pure strategies $\left(Z_{i}\right)_{i \in I}$ is considered finite. Tensor form updates the normal form which is limited in the representation of games, and doesn't have proper tools to use in many game theory problems, such as equilibrium problems. Moreover, its representation of games is not clear enough for $n>3$ players, mostly if the number of strategies is too large. Therefore, the introduced form based on tensors, that are mathematically defined using multi-linear algebra solves all these problems, rearrange games' characteristics (players, strategies, payoffs) and give mathematical access to games data. Tensor form provides also efficient methods to search for multiple equilibria, mostly in high games dimensions when the number of players is big. This problematic is rarely found in the literature of game theory but is solved using tensor form. All these advantages will be demonstrated below using definitions and examples. First, we start by defining tensors and giving some tensors' properties and operations.

\subsection{Tensors' properties}

In order to give the definitions and properties of the tensors, we adopt the definitions in $[19,20]$. In these papers, all technical properties of tensors are presented. In this paper, we tailor these properties to the game theory field. In $[19,20]$, tensors are defined respectively as arrays with different sizes and as multidimensional arrays. An order of a tensor is the number of its dimensions or also the number of its modes. For example, 


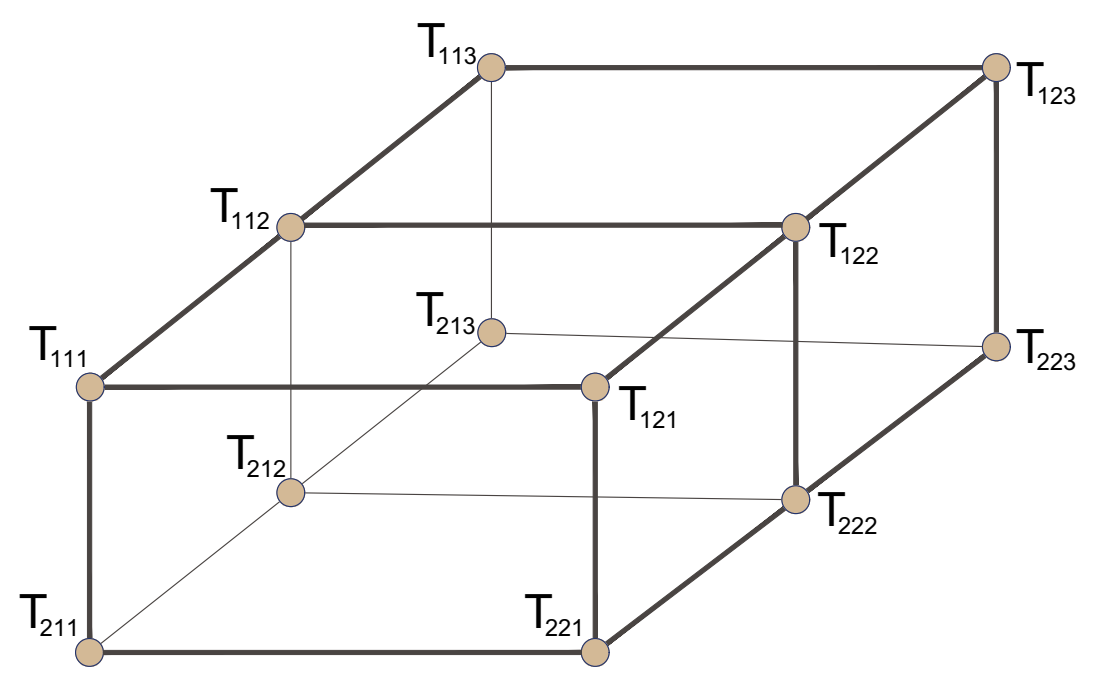

Figure 1. A third order tensor, where $d_{1}=2, d_{2}=2, d_{3}=3$.

the matrix $A \in M_{2,3}(\mathbb{R})$ is a 2 nd-order tensor. We write $A \in \mathbb{R}^{2 \times 3}$, it contains $2 \times 3=6$ elements. A 3rdorder tensor is denoted by $T \in \mathbb{R}^{2 \times 3 \times 2}$ and is shown in Figure 1. A $n$ th-order tensor $T$ is a multidimensional array in $\mathbb{R}^{d_{1} \times d_{2} \times \ldots \times d_{n}}$, which can be written as $T:=\left(T_{i_{1} i_{2} \ldots i_{n}}\right)$, where each element $T_{i_{1} i_{2} \ldots i_{n}} \in \mathbb{R}$, for any $i_{k} \in\left\{1, \ldots, d_{k}\right\}$, with $k \in\{1, \ldots, n\}$. The number of its elements is then $d_{1} \times d_{2} \times \ldots \times d_{n}$.

\subsubsection{Subarrays}

Subarrays of a $n$ th-order tensor $T$ are obtained by fixing a subset of indices of $T$. When fixing all indices but varying just one we obtain a subarray called fiber. For example, a Mode -1 fiber can be obtained by varying the first index of $T$ and fixing all others. It is denoted $T_{: i_{2} \ldots i_{n}}$. When all indices are fixed, but just two are varied, we obtain a subarray called slice. For example, a slice of $T$ can be obtained by fixing all indices but by varying the first two indices of $T$. We note this slice $T_{:: i_{3} i_{4} \ldots i_{n}}$. In the following, using Figure 1, we give examples of sketched fibers and slices of $T$ for $n=3$, see Figures 2 and 3.

\subsection{Tensor form of a game $\Gamma_{n}$}

Definition 2.1. Let $\Gamma_{n}=\left\langle I,\left(Z_{i}\right)_{i \in I},\left(p_{i}\right)_{i \in I}\right\rangle$ be a game. The tensor form of $\Gamma_{n}$ is given by $n$ tensors, denoted $T^{(i)}$, where $i \in\{1, \ldots, n\}$, for $n$ players. The elements of each tensor $T^{(i)}$ are defined by: $T_{j_{1} j_{2} \ldots j_{n}}^{(i)}=$ $p_{i}\left(z_{1}^{j_{1}}, z_{2}^{j_{2}}, \ldots, z_{i}^{j_{i}}, \ldots, z_{n}^{j_{n}}\right), \forall i \in\{1, \ldots, n\}$, for $j_{i} \in\left\{1,2, \ldots, \operatorname{card}\left(Z_{i}\right)\right\}$, where we note that $\left|Z_{i}\right|=\operatorname{card}\left(Z_{i}\right)$ and $T^{(i)} \in \mathbb{R}^{|Z|}$, such that $|Z|=\operatorname{card}\left(Z_{1}\right) \times \operatorname{card}\left(Z_{2}\right) \times \ldots \times \operatorname{card}\left(Z_{i}\right) \times \ldots \times \operatorname{card}\left(Z_{n}\right)$.

In this definition, $j_{1}, j_{2} \ldots, j_{n}$ represent at the same time a node coordinate and the profile of strategies $\left(z_{1}^{j_{1}}, z_{2}^{j_{2}}, \ldots, z_{i}^{j_{i}}, \ldots, z_{n}^{j_{n}}\right)$ chosen by the $n$ players. Therefore, in tensor form, each profile of strategies corresponds to a node coordinate. Indeed, these $n$ tensors can be merged in a multi-tensor $\mathcal{T} \in \mathbb{R}^{|Z|}$ by writing at each node of $\mathcal{T}$ the whole utility function $p=\left(p_{1}, p_{2}, \ldots, p_{n}\right)$ instead, of a simple payoff $p_{i}$. This concept is used in bimatrices of normal form in a 2-player game. In the following, we separate between the node coordinates yielding the profile of strategies and the components of the node value containing the payoff of each player. For instance, in a 3 -player game, $\mathcal{T}_{111}=(-1,5,9)$ represents a node (element) having the coordinates $(1,1,1)$. However, the components of its node value are the payoffs $-1,5$ and 9 for the three players respectively. Consequently, we see that tensor form rearrange all characteristics of game $\Gamma_{n}$ in the multi-tensor $\mathcal{T}$. 


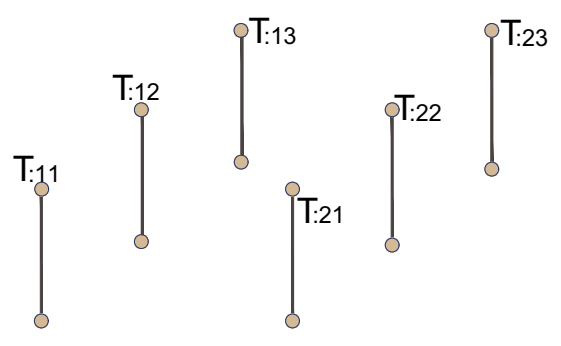

(a)

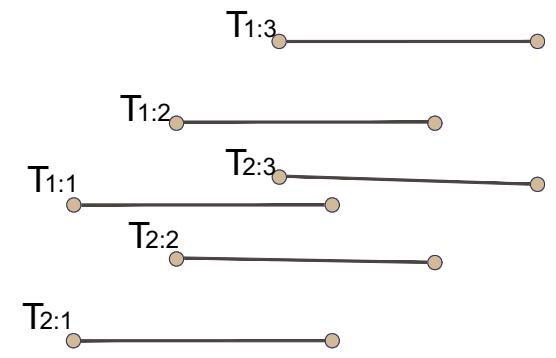

(b)

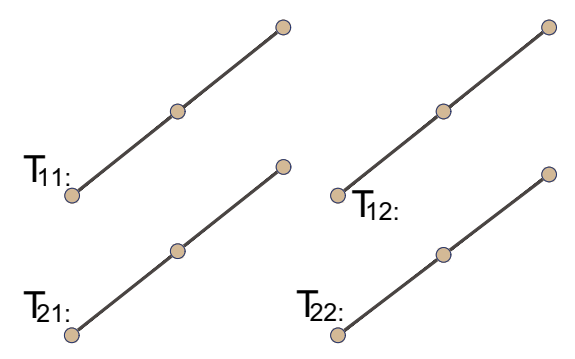

(c)

Figure 2. Mode -1 , Mode -2 and Mode -3 fibers of T. (a) Column (Mode -1 ) fibers. (b) Row (Mode -2 ) fibers. (c) Tube (Mode -3 ). fibers.

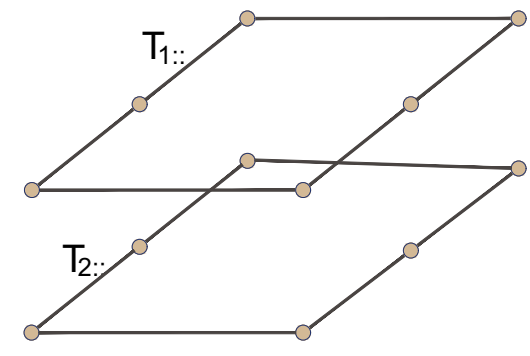

(a)

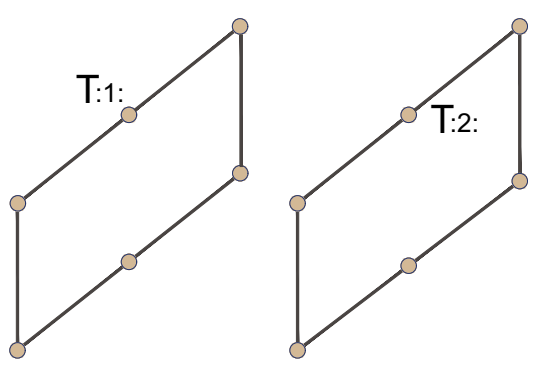

(b)

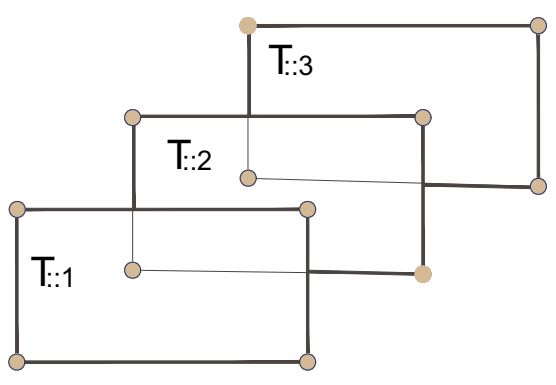

(c)

Figure 3. Horizontal, Lateral and Frontal slices of $T$. (a) Horizontal slices. (b) Lateral slices. (c) Frontal slices. 


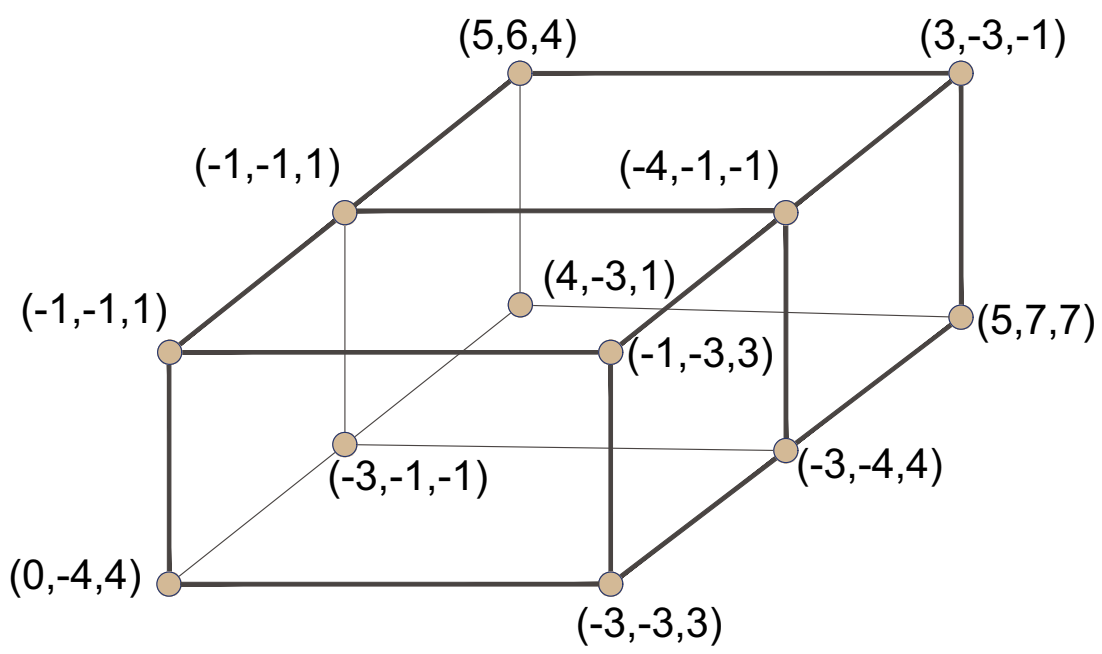

Figure 4. Tensor form of $\Gamma_{3}$.

\subsubsection{Numerical example for a tensor form}

We consider a game $\Gamma_{3}=\left\langle I=\{1,2,3\},\left(Z_{1}, Z_{2}, Z_{3}\right),\left(p_{1}, p_{2}, p_{3}\right)\right\rangle$, where, $Z_{1}=\left\{z_{1}^{1}, z_{1}^{2}\right\}, Z_{2}=\left\{z_{2}^{1}, z_{2}^{2}\right\}$ and $Z_{3}=\left\{z_{3}^{1}, z_{3}^{2}, z_{3}^{3}\right\}$. According to Definition 2.1, the tensor form of $\Gamma_{3}$ is given by three tensors $T^{(i)} \in \mathbb{R}^{2 \times 2 \times 3}$, where $T^{(i)}=p_{i}\left(z_{1}, z_{2}, z_{3}\right)$ for $i \in\{1,2,3\}$. Indeed, these tensors can be merged in a tensor $\mathcal{T} \in \mathbb{R}^{2 \times 2 \times 3}$ represented geometrically in the Figure 4 . Here, each node in $\mathcal{T}$ represents a utility function composed of three payoffs. For example, if "the first player chooses the strategy $z_{1}^{1}$, the second player chooses the strategy $z_{2}^{1}$ and the third player chooses the strategy $z_{3}^{\mathbf{3}}$ ". This particular strategies profile is then represented by the node $\mathcal{T}_{\mathbf{1 1 3}}$. Here, $\mathcal{T}_{\mathbf{1 1 3}}=\left(p_{1}\left(z_{1}^{1}, z_{2}^{1}, z_{3}^{3}\right), p_{2}\left(z_{1}^{1}, z_{2}^{1}, z_{3}^{3}\right), p_{3}\left(z_{1}^{1}, z_{2}^{1}, z_{3}^{3}\right)\right)=(5,6,4)$.

\section{TENSOR FORM OF GAMES AND EQUILIBRIA}

Let $\Gamma_{n}=\left\langle I,\left(Z_{i}\right)_{i \in I},\left(p_{i}\right)_{i \in I}\right\rangle$ be a non-cooperative game with $n$ players. This game is parametrized by the set of players $I=\{1, \ldots, i, \ldots n\} \subset \mathbb{N}$, a set of pure strategies $Z_{i}$ and the Von-Neumann-Morgenstern utility function $p_{i}$ for every player $i \in I$. In addition, we note $z=\left(z_{1}, \ldots, z_{i}, \ldots, z_{n}\right)$ a profile of pure strategies, where $z \in Z=\underset{i=1}{n} Z_{i}$ with $z_{-i}=\left(z_{1}, \ldots, z_{i-1}, z_{i+1}, \ldots, z_{n}\right)$ is an incomplete profile of pure strategies from players other than $i$, where $z_{-i} \in Z_{-i}=\underset{\substack{j \neq i \\ j}}{\times} Z_{j}$. In general, equilibrium types are attached to players' behavior, see [8]. In this section, we write the definition of three equilibrium types. We start by defining pure Nash equilibrium and pure Berge equilibrium. We briefly recall as well their formulas using tensor form, shown respectively in $[3,29]$, a prior to defining pure unilateral support equilibrium and its tensor form. The objective is to situate the idea of the unilateral support equilibrium within Nash and Berge concepts, know the relations between them, locate them on the tensor, and highlight the differences in the equilibrium search procedures of these three equilibria. We use the same example 2.2.1 for illustrations. The study focuses on the field of non-cooperative games. Moreover, we propose a novel method to search for pure USE using tensors tools called fibers for any number of players $n \in \mathbb{N}$. These fibers have the advantage of geometrical representation for $n=3$.

\subsection{Tensor form applied to Nash equilibrium}

At first hand, we begin with Nash equilibrium, which is provided by an individualistic orientation of the players. Each player only is supported by himself. 
Definition 3.1. A pure strategy profile $z^{\star} \in Z$ is a pure Nash equilibrium of game $\Gamma_{n}$ if,

$$
\forall i \in I, \quad p_{i}\left(z_{-i}^{\star}, z_{i}^{\star}\right) \geq p_{i}\left(z_{-i}^{\star}, \quad z_{i}\right) ; \forall z_{i} \in Z_{i} .
$$

It is clear that each player $i$ plays individualistically a strategy among his own strategies $z_{i} \in Z_{i}$ in order to maximize his own payoff $p_{i}$. He supports, and is as well, supported, only by himself.

This definition is also written using tensor form in [3] as follows: a pure strategy profile $z^{\star}=$ $\left(z_{1}^{k_{1}^{*}}, \ldots, z_{i}^{k_{i}^{*}}, \ldots, z_{n}^{k_{n}^{*}}\right) \in Z$ is a pure Nash equilibrium of game $\Gamma_{n}$ if,

$$
\forall i \in\{1,2, \ldots, n\} T_{k_{1}^{*} k_{2}^{*} \ldots k_{i}^{*} \ldots k_{n}^{*}}^{(i)} \geq T_{k_{1}^{*} k_{2}^{*} \ldots k_{i} \ldots k_{n}^{*}}^{(i)} .
$$

Interpretation: in the second member $T_{k_{1}^{*} k_{2}^{*} \cdot k_{i} \ldots k_{n}^{*}}^{(i)}$ all indices of the tensor are fixed, except $k_{i}$. Thus, when we search for all $k_{i}$ it means that we are going along the Mode $-i$ fiber passing by the Nash equilibrium candidate node. In fact, each player $i$, is searching for a strategy that maximizes his own payoff. Therefore, we can understand inequality (3.2) as follows: for each node candidate to be a Nash equilibrium, we fix a player $i$ (the node value component number $i$ in the multi-tensor $\mathcal{T}$ ).

Then, we search for all Mode - $i$ fibers passing by this candidate node. We compare then in each Mode $i$ fiber the nodes values components number $i$ in the multi-tensor $\mathcal{T}$. If the node value component number $i$ in the node candidate to Nash equilibrium, is greater than all other nodes values components number $i$ in the multi-tensor $\mathcal{T}$, on the Mode $-i$ fiber, then we can pass to another player $i$. These inequalities must hold for all $i$ and then for all the coordinates of the candidate node. Therefore, inequality (3.2) will be transformed to comparison inside fibers instead of writing all inequalities holding Definition 3.1. Hereinafter, this tensor searching method for Nash equilibrium will be used in the following numerical example.

Example: We search here for pure Nash equilibrium in the same example 2.2.1. Let's start by searching for a profile of strategies verifying the concept of Nash equilibrium. Instead of writing all inequalities verifying the Nash equilibrium in Definition 3.1, we can proceed by using tensor form. For example, we handle the node having the coordinates $(1,1,3)$ on $\mathcal{T}$, corresponding to the profile $\left(z_{1}^{1}, z_{2}^{1}, z_{3}^{3}\right)$. Its utility is $(5,6,4)$. The first step consists of extracting the node with its intersected fibers, see Figure 8 . Then, by applying the concept of tensor inequalities defined in inequality (3.2), and the previous tensor searching method we can see that the following comparisons through fibers are true: see Figure 5 a for player $i=1$, here the proposition $5>4$ is true.

See Figure $5 \mathrm{~b}$ for player $i=2$, here the proposition $6>-3$ is true.

See Figure 5 c for player $i=3$, here the proposition $(4>1)$ and $(4>1)$ is true.

Therefore, since all comparisons through fibers are true, we conclude that the node $\mathcal{T}_{113}$ corresponding to the profile $\left(z_{1}^{1}, z_{2}^{1}, z_{3}^{3}\right)$ is a pure Nash equilibrium. As already explained, every set of inequalities corresponding to a player $i$ in the classic definition of Nash equilibrium in Definition 3.1 is transformed to a comparison in a tensor's mathematical tool called fiber. And then, comparison through fibers can replace writing all inequalities to find a Nash equilibrium.

This game contains also a second Nash equilibrium. By extracting node $\mathcal{T}_{223}$, see Figure 10, and following the same procedure as above we can also verify that the profile of strategies $\left(z_{1}^{2}, z_{2}^{2}, z_{3}^{3}\right)$ corresponding to this node is a pure Nash equilibrium. The figures corresponding to the procedure of finding pure Nash equilibrium for this node are shown in $6 \mathrm{a}-6 \mathrm{c}$.

\subsection{Tensor form applied to Berge equilibrium}

Berge equilibrium is provided by mutual support between players motivated by the altruistic social value orientation. Each player is supported by the group of all other players in the game, see [26,33]. This equilibrium is also called Berge-Zhukovskii equilibrium (see [11] for several definitions and refinements of Berge equilibrium).

Definition 3.2. A pure strategy profile $z^{\star} \in Z$ is a pure Berge equilibrium of game $\Gamma_{n}$ if,

$$
\forall i \in I, \quad p_{i}\left(z_{-i}^{\star}, z_{i}^{\star}\right) \geq p_{i}\left(z_{-i}, z_{i}^{\star}\right) ; \forall z_{-i} \in Z_{-i} .
$$




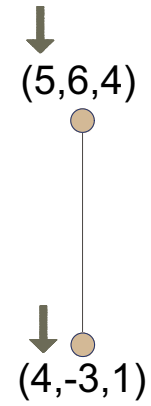

(a)

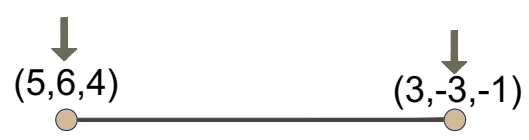

(b)

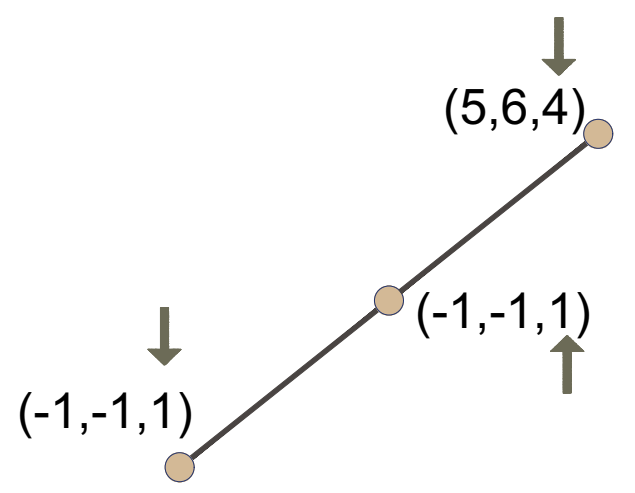

(c)

Figure 5. Fibers of Node $\mathcal{T}_{113}$. (a) Mode -1 fiber of node $\mathcal{T}_{113}$. (b) Mode -2 fiber of node $\mathcal{T}_{113}$. (c) Mode -3 fiber of the node $\mathcal{T}_{113}$.

It is clear that the group of $n-1$ players of the game, without player $i$, choose among their incomplete profile $z_{-i} \in Z_{-i}$ an incomplete profile that maximizes $p_{i}$ the payoff of player $i$. Here, the $n-1$ players, without $i$, support all together player $i$, and this behavior is mutual for all players. It is called altruistic.

In [29], we can find this definition written using tensor form as follows: a pure strategy profile $z^{\star}=$ $\left(z_{1}^{k_{1}^{*}}, \ldots, z_{i}^{k_{i}^{*}}, \ldots, z_{n}^{k_{n}^{*}}\right) \in Z$ is a pure Berge equilibrium of game $\Gamma_{n}$ if,

$$
T_{k_{1}^{*} k_{2}^{*} \ldots k_{i}^{*} \ldots k_{n}^{*}}^{(i)}>T_{k_{1} k_{2} \ldots k_{i}^{*} \ldots k_{n}}^{(i)}, \forall i \in\{1,2, \ldots, n\}
$$

Interpretation: in the second member $T_{k_{1} k_{2} \ldots k_{i}^{*} \ldots k_{n}}^{(i)}$, all indices of the tensor are varying except $k_{i}^{*}$, only $k_{i}^{*}$ is fixed. Thus, we search here for all incomplete profiles $\left(k_{1} k_{2} \ldots k_{i-1} k_{i+1} \ldots k_{n}\right)$, which maximize player $i$ 's payoff. It means that we are going along all subarrays $T: \ldots: k_{i}^{\star}: \ldots$ : intersecting the candidate node, for all $i=1, \ldots, n$. In each subarray, the $i$ th node value component of the candidate node must be greater than the $i$ th node value component of all nodes in the subarray $T: \ldots: k_{i}^{\star}: \ldots$ : and that is true for all $i=1, \ldots, n$. Therefore, inequality (3.4) will be transformed to comparison inside subarrays instead of writing all inequalities holding Definition 3.2. This tensor searching method for Berge equilibrium will be used in the following numerical example.

Example: We will search now for Berge equilibrium in the same example 2.2.1. When we apply the tensor procedure to search for such a profile, we can find that we only have one Berge equilibrium which is in the node $\mathcal{T}_{223}$. It corresponds to the profile $\left(z_{1}^{2}, z_{2}^{2}, z_{3}^{3}\right)$ of utility $(5,7,7)$. The first step consists of considering the three subarrays intersected in the candidate node. They are $\mathcal{T}_{2::}, \mathcal{T}_{: 2:}, \mathcal{T}_{:: 3}$, see Figures $7 \mathrm{a}-7 \mathrm{c}$ respectively. Then, by applying the concept of tensor inequalities defined in inequality (3.4), and the previous tensor searching 


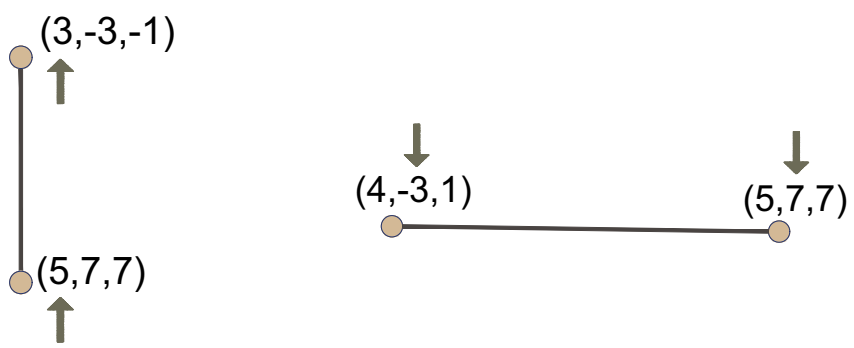

(a)

(b)

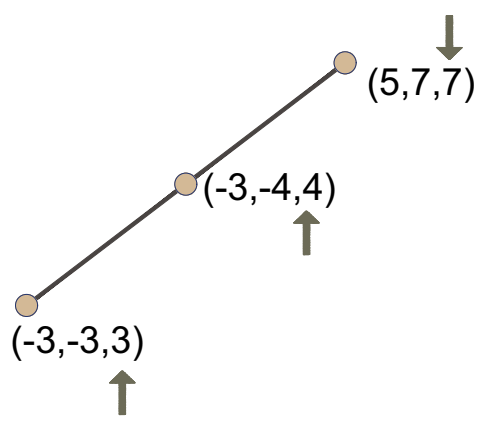

(c)

Figure 6 . Fibers of Node $\mathcal{T}_{223}$. (a) Mode -1 fiber of node $\mathcal{T}_{223}$. (b) Mode -2 fiber of node $\mathcal{T}_{223}$. (c) Mode -3 fiber of node $\mathcal{T}_{223}$.

method, we can see that the following comparisons through fibers are true: see Figure 7a for player $i=1$, here the proposition $(5>-3) \&(5>0) \&(5>4)$ is true.

See Figure $7 \mathrm{~b}$ for player $i=2$, here the proposition $(7>-4) \&(7>-3) \&(7>-1)$ is true.

See Figure $7 \mathrm{c}$ for player $i=3$, here the proposition $(7>-1) \&(7>1) \&(7>4)$ is true.

Therefore, since all comparisons through subarrays are true, we conclude that the node $\mathcal{T}_{223}$ corresponding to the profile $\left(z_{1}^{2}, z_{2}^{2}, z_{3}^{3}\right)$ is a pure Berge equilibrium. As demonstrated in this tensor procedure, every set of inequalities corresponding to a player $i$ in the classic definition of Berge equilibrium in Definition 3.2 is changed to a comparison in a tensor's mathematical tool called subarray. Subsequently, comparison through subarrays can replace writing all inequalities to find a Berge equilibrium.

\subsection{Unilateral support equilibria and tensor form}

Unilateral support equilibrium is provided when every player is supported by every other player individually; see [30].

Definition 3.3. A pure strategy profile $z^{\star} \in Z$ is a pure unilateral support equilibrium of game $\Gamma_{n}$ if,

$$
\forall i \in I, \forall j \in I \backslash\{i\}, p_{i}\left(z_{-j}^{\star}, z_{j}^{\star}\right) \geq p_{i}\left(z_{-j}^{\star}, z_{j}\right) ; \forall z_{j} \in Z_{j} .
$$

We note that each player $i$ is supported by a player $j$ individually for each $j \neq i$. Player $j$ chooses among his pure strategies $z_{j} \in Z_{j}$ a strategy maximizing the payoff $p_{i}$ of player $i$. In the following, we will abbreviate the unilateral support equilibrium by (USE).

To clarify these definitions we consider a game $\Gamma_{3}=\left\langle I=\{1,2,3\},\left(Z_{1}, Z_{2}, Z_{3}\right),\left(p_{1}, p_{2}, p_{3}\right)\right\rangle$, where, $Z_{1}=$ $\left\{z_{1}^{1}, z_{1}^{2}\right\}, Z_{2}=\left\{z_{2}^{1}, z_{2}^{2}\right\}$ and $Z_{3}=\left\{z_{3}^{1}, z_{3}^{2}\right\}$, illustrated in the following Table 1 in classic normal form: 


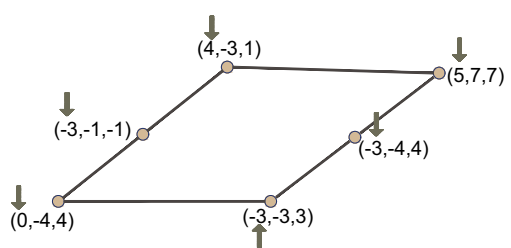

a)

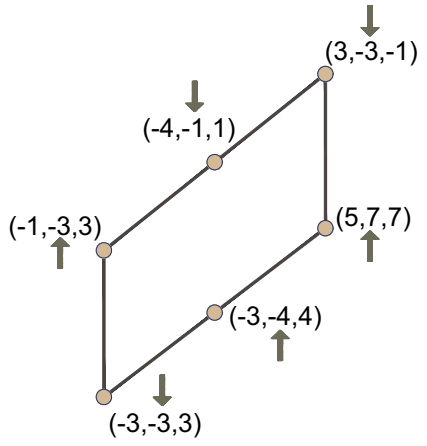

(b)

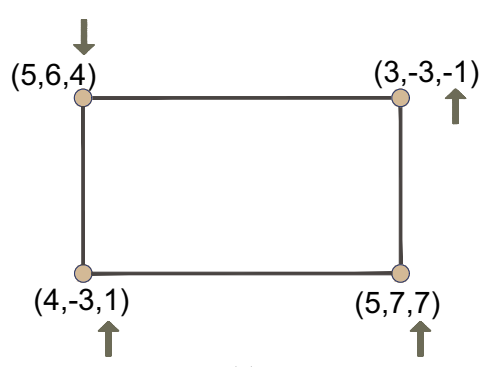

(c)

FIgURE 7. Subarrays (slices) intersected in node $\mathcal{T}_{223}$. (a) Subarray $\mathcal{T}_{2: \text { : }}$ of node $\mathcal{T}_{223}$. (b) Subarray $\mathcal{T}_{\text {:2: }}$ of node $\mathcal{T}_{223}$. (c) Subarray $\mathcal{T}_{:: 3}$ of node $\mathcal{T}_{223}$.

TABLE 1. 3-player game with two strategies each one.

\begin{tabular}{lll}
\hline \hline$z_{3}^{1}$ & $z_{2}^{1}$ & $z_{2}^{2}$ \\
\hline$z_{1}^{1}$ & $(1,1,0)$ & $(0,0,0)$ \\
$z_{1}^{2}$ & $(0,0,0)$ & $(0,0,1)$ \\
\hline
\end{tabular}

\begin{tabular}{lll}
\hline \hline$z_{3}^{2}$ & $z_{2}^{1}$ & $z_{2}^{2}$ \\
\hline$z_{1}^{1}$ & $(0,0,0)$ & $(0,0,0)$ \\
$z_{1}^{2}$ & $(0,0,0)$ & $(1,1,0)$ \\
\hline
\end{tabular}

By using Definitions 3.1-3.3, it is shown that the profile $\left(z_{1}^{1}, z_{2}^{1}, z_{3}^{1}\right)$ is a USE (and also a Nash equilibrium) but not a Berge equilibrium. This simple example gives an preliminary illustration of the difference between USE and Berge equilibria in the sense that every Berge equilibrium is necessary an USE but the inverse is not always true.

Let us recall two useful notions for finding Nash and Berge equilibrium. First of all, the set of Best-Response of a player $i$ to a fixed incomplete profile strategies $z_{-i}$ is $\mathrm{BR}_{i}\left(z_{-i}\right)=\left\{z_{i} \in Z_{i}, p_{i}\left(z_{-i}, z_{i}\right) \geq p_{i}\left(z_{-i}, z_{i}^{\prime}\right), \forall z_{i}^{\prime} \in Z_{i}\right\}$, see [28]. Using this set, we can write Definition 3.1 as:

A pure strategy profile $z^{\star} \in Z$ is a pure Nash equilibrium of game $\Gamma_{n}$ if, and only if, $\forall i \in I, z_{i}^{\star} \in \mathrm{BR}_{i}\left(z_{-i}^{\star}\right)$.

Secondly, the set of Best Support from co-players $-i$ to a strategy $z_{i}$ of player $i$, introduced in [23] is $\mathrm{BS}_{i}\left(z_{i}\right)=\left\{z_{-i} \in Z_{-i}, p_{i}\left(z_{-i}, z_{i}\right) \geq p_{i}\left(z_{-i}^{\prime}, z_{i}\right), \forall z_{-i}^{\prime} \in Z_{-i}\right\}$. Using this set, we can write Definition 3.2 as:

A pure strategy profile $z^{\star} \in Z$ is a pure Berge equilibrium of game $\Gamma_{n}$ if, and only if, $\forall i \in I, z_{-i}^{\star} \in \operatorname{BS}_{i}\left(z_{i}^{\star}\right)$.

Similarly, we introduce a new set dedicated to unilateral support equilibrium. 
Definition 3.4. The set of Best Individualistic Support to player $i$, offered by player $j$, given a fixed incomplete profile of pure strategies $z_{-j}$ is defined by:

$$
\operatorname{BIS}_{i, j}\left(z_{-j}\right)=\left\{z_{j} \in Z_{j} ; p_{i}\left(z_{-j}, z_{j}\right) \geq p_{i}\left(z_{-j}, z_{j}^{\prime}\right), \forall z_{j}^{\prime} \in Z_{j}\right\} .
$$

Using this set, we can write Definition 3.3 as:

A pure strategy profile $z^{\star} \in Z$ is a pure USE of game $\Gamma_{n}$ if, and only if, $\forall i \in I, \forall j \in I \backslash\{i\}, z_{j}^{\star} \in$ $\operatorname{BIS}_{i, j}\left(z_{-j}^{\star}\right)$.

In this section, we use tensor form to write the USE inequalities using tensors. The tensor form of USE gives a mathematical aspect to USE and will provides a method to search for pure USE using some tools of tensors called fibers for any number of players $n \in \mathbb{N}$. These fibers have the advantage of geometrical representation for $n=3$. Let's start by the game $\Gamma_{n}=\left\langle I,\left(Z_{i}\right)_{i \in I},\left(p_{i}\right)_{i \in I}\right\rangle$. In Definition 3.3, we said that a pure strategy profile $z^{\star} \in Z$ is a pure USE of game $\Gamma_{n}$ if,

$$
\forall i \in I, \forall j \in I \backslash\{i\}, p_{i}\left(z_{-j}^{\star}, z_{j}^{\star}\right) \geq p_{i}\left(z_{-j}^{\star}, z_{j}\right) ; \forall z_{j} \in Z_{j} .
$$

This definition can be written using tensors which will provide us to take some advantages to find the USE using tensors' tools and operations as follows: a profile $z^{*}=\left(z_{1}^{k_{1}^{*}}, \ldots, z_{i}^{k_{i}^{*}}, \ldots, z_{n}^{k_{n}^{*}}\right)$, corresponding to the node having the coordinates $k_{1}^{*}, \ldots k_{i}^{*}, \ldots, k_{n}^{*}$ in the multi-tensor $\mathcal{T}$ (gathering all tensors $T^{(i)}$ ), is a pure USE if,

$$
\forall i \in I, \forall j \in I \backslash\{i\}, \quad T_{k_{1}^{*} \ldots k_{i}^{*} \ldots k_{j}^{*} \ldots k_{n}^{*}}^{(i)} \geq T_{k_{1}^{*} \ldots k_{i}^{*} \ldots k_{j} \ldots k_{n}^{*}}^{(i)}, \forall k_{j} \in\left\{1, \ldots, \operatorname{card}\left(Z_{j}\right)\right\} .
$$

In the second member $T_{k_{1}^{*} \ldots k_{i}^{*} \ldots k_{j} \ldots k_{n}^{*}}^{(i)}$, all indices of the tensor are fixed except $k_{j}$. Thus, when we search for all $k_{j}$ it means that we are going along the Mode $-j$ fiber passing by the USE candidate node. Therefore, inequality (3.6) is explained as follows: for each node candidate to be USE, we fix a player $i$ (the coordinate number $i$ in the multi-tensor $\mathcal{T}$ ). Then, we search in all Mode $-j$ fibers passing by this node without Mode $i$ fiber. We compare then in each Mode - $j$ fiber the nodes values components number $i$ in the multi-tensor $\mathcal{T}$. If the node value component number $i$ in the node candidate to be USE, is greater than all other nodes values components number $i$ in the multi-tensor $\mathcal{T}$, on the Mode $-j$ fibers, we can then pass to another player $i$. These inequalities must hold for all $i$ and then for all the coordinates of the candidate node. Therefore, inequality (3.6) will be transformed to comparison inside fibers instead of writing all inequalities holding Definition 3.3. This principle will give a big advantage to programmers to search for USE using their proper algorithms, knowing that tensor class is given by [20] in [4]. This idea is demonstrated by the following 3-player game in order to present it geometrically. In the following, this tensor searching method for USE will be used in all our numerical examples.

\subsection{Numerical Example for a pure USE with tensors}

We consider the same game $\Gamma_{3}=\left\langle I=\{1,2,3\},\left(Z_{1}, Z_{2}, Z_{3}\right),\left(p_{1}, p_{2}, p_{3}\right)\right\rangle$ in Section 2.2.1, where, $Z_{1}=\left\{z_{1}^{1}, z_{1}^{2}\right\}$, $Z_{2}=\left\{z_{2}^{1}, z_{2}^{2}\right\}$ and $Z_{3}=\left\{z_{3}^{1}, z_{3}^{2}, z_{3}^{3}\right\}$. Tensor form is given in Figure 4. Now, we have to search for a profile of strategies verifying a USE. Instead of writing all inequalities of USE in Definition 3.3 and searching for all $i$ and $j$, we can proceed by using tensor form. For example, we handle the node $\mathcal{T}_{113}$, corresponding to the profile $\left(z_{1}^{1}, z_{2}^{1}, z_{3}^{3}\right)$. Its utility is $(5,6,4)$. The first step consists of extracting the node with its intersected fibers, see Figure 8 .

Then, by applying the concept of tensor's inequalities defined in inequality (3.6), and the previous tensor searching method we can see that the following comparisons through fibers are true:

see Figure 9a for player $i=1$, here the proposition $(5>-1)$ and $(5>-1)$ and $(5>3)$ is true, see Figure $9 \mathrm{~b}$ for player $i=2$, here the proposition $(6>-1)$ and $(6>-1)$ and $(6>-3)$ is true, see Figure $9 \mathrm{c}$ for player $i=3$, here the proposition $(4>-1)$ and $(4>1)$ is true. 


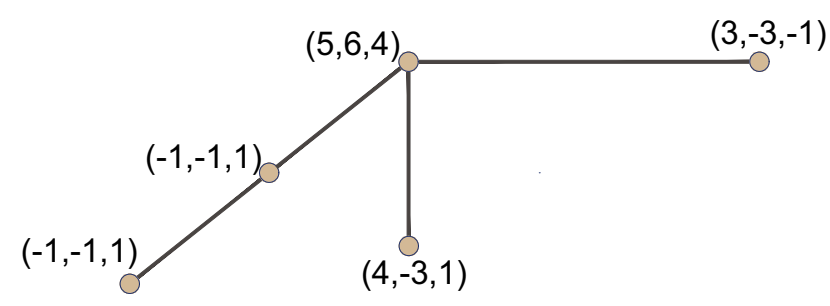

FiguRE 8 . Extraction of node $\mathcal{T}_{113}$ with its fibers.

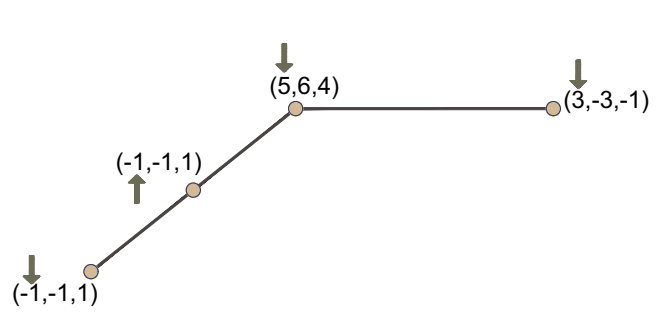

(a)

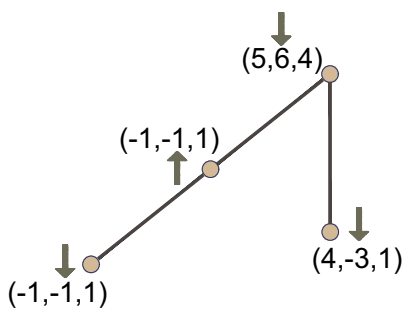

(b)

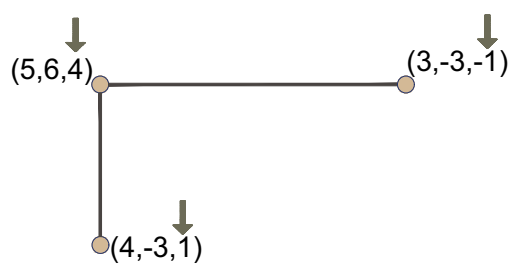

(c)

FiguRE 9. Intersecting fibers in node $\mathcal{T}_{113}$ without player $i$ fibers, respectively for $i=1,2,3$. (a) Intersecting fibers in node $\mathcal{T}_{113}$ without Mode -1 fiber. (b) Intersecting fibers in node $\mathcal{T}_{113}$ without Mode -2 fiber. (c) Intersecting fibers in node $\mathcal{T}_{113}$ without Mode -3 fiber.

We conclude that the node $\mathcal{T}_{113}$ corresponding to the profile $\left(z_{1}^{1}, z_{2}^{1}, z_{3}^{3}\right)$ is a pure USE. According to this tensor procedure, every set of inequalities corresponding to a player $i$ in the classic definition of USE in Definition 3.3 is changed by a comparison in a tensor's mathematical tool called fiber. Afterward, comparison through fibers can replace writing all inequalities to find a USE.

Moreover, we handle the node, having the coordinates $(2,2,3)$. By extracting this node, see Figure 10, and following the same procedure as above, we can verify that the profile of strategies $\left(z_{1}^{2}, z_{2}^{2}, z_{3}^{3}\right)$ corresponding to this node is a pure USE. The figures corresponding to the procedure of finding USE for this node are shown in $11 \mathrm{a}-11 \mathrm{c}$. However, we can also prove using the same procedure that this example possesses only these two computed pure USE.

\subsection{Relations between equilibria}

(1) In [30], the authors prove that each Berge equilibrium is a USE. This result appears clearly when taking the tensor form of Berge equilibrium and more precisely when we search on the candidate node through slices. If Berge equilibrium is reached on these slices then the fibers corresponding to USE same node candidate are covered. This is illustrated on the node $\mathcal{T}_{223}$ corresponding slices and fibers. Moreover, the reciprocal is false as seen on tensors, for $n>2$. In other words, a USE is not necessary a Berge equilibrium. 


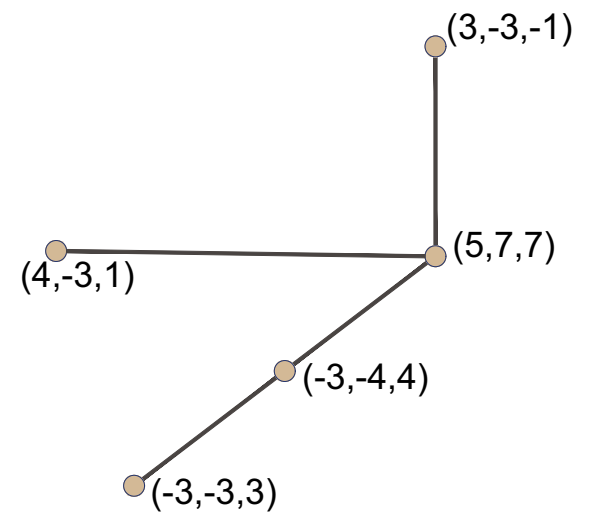

FIgURE 10. Extraction of node $\mathcal{T}_{223}$ with its fibers.
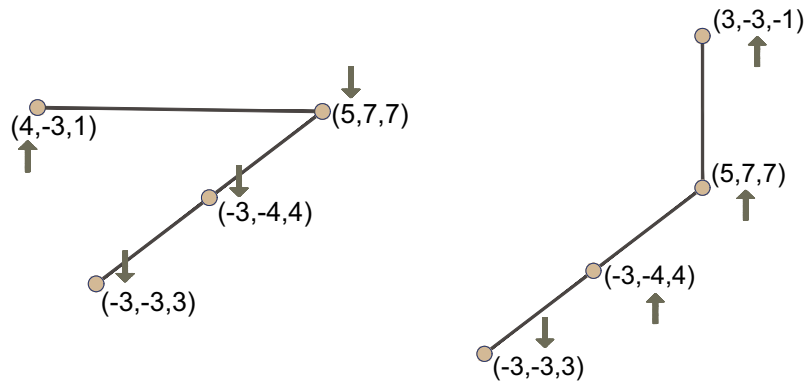

(a)

(b)

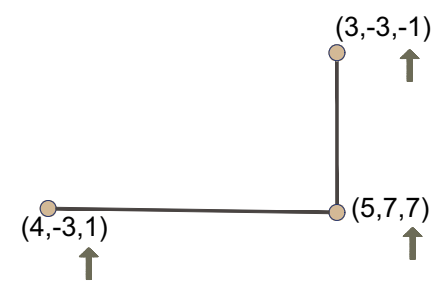

(c)

FiguRE 11. Extraction of Node $\mathcal{T}_{223}$ with its fibers, except those of player $i$, respectively for $i=1,2,3$. (a) Intersecting fibers in node $\mathcal{T}_{223}$ without Mode -1 fiber. (b) Intersecting fibers in node $\mathcal{T}_{223}$ without Mode -2 fiber. (c) Intersecting fibers in node $\mathcal{T}_{223}$ without Mode -3 fiber.

(2) In Definition 3.1, we explained the selfish behavior of players. In Definition 3.2, we talked about altruistic mutual support between players that took many years to be elaborated from the initial definition. In Definition 3.3, we explained that each player $i$ is supported by a player $j$ individually, for each $j \neq i$. All these interpretations are seen on tensors without any difficulties by individualistic fibers for Nash, other players slices for Berge, and other players fibers' for USE. 


\section{Algorithm to Find pure USE Using tensor FOrm}

We present in this section an algorithm based on tensors as main variables, using tensor form of a game in order to compute USE problems for games where $n \geq 3$ players. In these games, we lose the advantage of visualizing tensors geometrically. Despite this lost, all advantages of tensors are well preserved. In fact, the concept of searching for fibers and using tensor inequality (3.6) will solve the problem. The efficiency is shown when computing a pure USE with high dimensional problems. We show here, in an example, a direct application by solving a game of 15 players. The complexity of this algorithm is $I_{1} \times I_{2} \times \ldots I_{n}$, see Algorithm 1 .

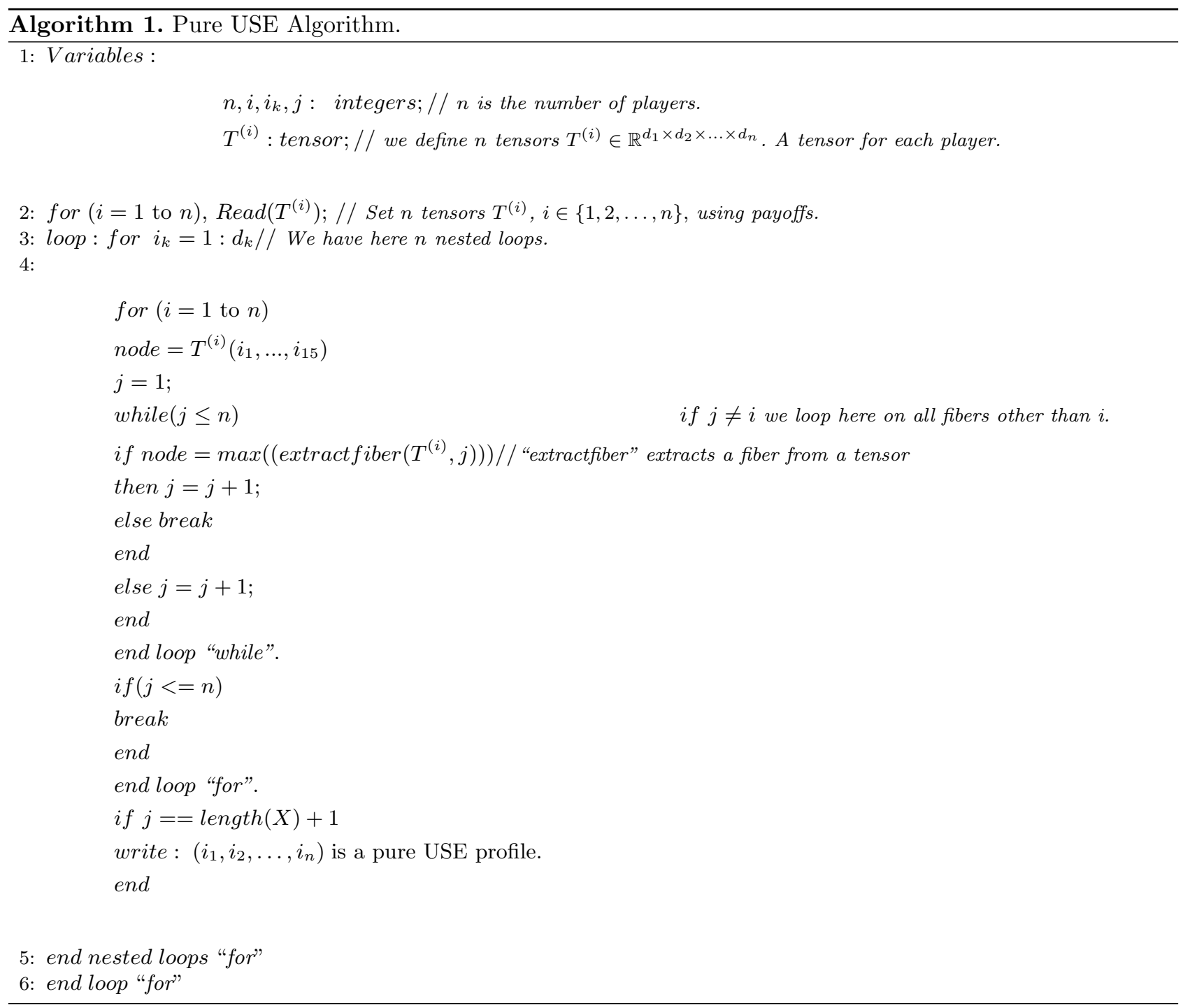

\subsection{Application to a High dimensional USE problem}

We consider a game $\Gamma_{15}=\left\langle I=\{1,2, \ldots, 15\},\left(Z_{1}, Z_{2}, \ldots, Z_{15}\right),\left(p_{1}, p_{2}, \ldots, p_{15}\right)\right\rangle$, where, $Z_{i}=\left\{z_{i}^{1}, z_{i}^{2}\right\}$, for all $i=1, \ldots, 15$. The tensor form of $\Gamma_{15}$ is given by 15 tensors $T^{(i)} \in \mathbb{R}^{2 \times 2 \times \ldots \times 2}$, where $T^{(i)}=p_{i}\left(z_{1}, z_{2}, \ldots, z_{15}\right)$ for $i \in\{1,2, \ldots, 15\}$. These tensors are merged in a multi-tensor $\mathcal{T} \in \mathbb{R}^{2 \times 2 \times \ldots \times 2}$. Each node in $\mathcal{T}$ represents a utility 
function composed of 15 payoffs given by $\mathcal{T}_{i_{1} i_{2} \ldots i_{15}}=-i_{1}-2 i_{2}-3 i_{3}-4 i_{4}-5 i_{5}+6 i_{6}+7 i_{7}+8 i_{8}+9 i_{9}-10 i_{10}+i_{11}+i_{12}+$ $i_{13}-i_{14}-i 15+e$ where $e$ is the number of tensor element going from 1 to $15 \times 2^{15}$. Using Algorithm 1, we can find that there are two pure USE. The first one in the node $\mathcal{T}_{2,2,2,2,2,2,2,2,2,2,2,2,2,2,1}=(32780,65548,98316,131084$, $163852,196620,229388,262156,294924,327692,360460,393228,425996,458764,491532)$ corresponding to the profile

$$
\left(z_{1}^{2}, z_{2}^{2}, z_{3}^{2}, z_{4}^{2}, z_{5}^{2}, z_{6}^{2}, z_{7}^{2}, z_{8}^{2}, z_{9}^{2}, z_{10}^{2}, z_{11}^{2}, z_{12}^{2}, z_{13}^{2}, z_{14}^{2}, z_{15}^{1}\right)
$$

and the second one in the node $\mathcal{T}_{2,2,2,2,2,2,2,2,2,2,2,2,2,2,2}=(32780,65548,98316,131084,163852$, $196620,229388,262156,294924,327692,360460,393228,425996,458764,491532)$ having the same utility as the first one due to the formula when we add $+k-i_{15}$. It corresponds to the profile

$$
\left(z_{1}^{2}, z_{2}^{2}, z_{3}^{2}, z_{4}^{2}, z_{5}^{2}, z_{6}^{2}, z_{7}^{2}, z_{8}^{2}, z_{9}^{2}, z_{10}^{2}, z_{11}^{2}, z_{12}^{2}, z_{13}^{2}, z_{14}^{2}, z_{15}^{2}\right)
$$

\section{Mixed Unilateral SUPPORT EQUilibria}

In this section, we present a lemma dedicated to calculate mixed USE profiles when the set of pure strategies is finite. Let $\Gamma_{n}=\left\langle I,\left(Z_{i}\right)_{i \in I},\left(\mathcal{Z}_{i}\right)_{i \in I},\left(p_{i}\right)_{i \in I}\right\rangle$ be a non-cooperative game with $n$ players, where $\mathcal{Z}_{i}$ is a set of mixed strategies. In addition, we note $z=\left(z_{1}, \ldots, z_{i}, \ldots, z_{n}\right)$ a profile of mixed strategies, where $z \in \mathcal{Z}=\stackrel{n}{\times} \mathcal{Z}_{i}$ with $z_{-i}=\left(z_{1}, \ldots, z_{i-1}, z_{i+1}, \ldots, z_{n}\right)$ is an incomplete profile of mixed strategies from players other than $i$, where $z_{-i} \in \mathcal{Z}_{-i}=\underset{j \neq i}{\times \mathcal{Z}_{j}}$. We start by giving some properties of mixed strategies.

(1) For any player $i \in I$, a mixed strategy, denoted by $z_{i} \in \mathcal{Z}_{i}$, is a probability distribution over this player pure strategies. Indeed, $z_{i}\left(z_{i}^{j}\right)$ is the probability that player $i$ plays the pure strategy $z_{i}^{j} \in Z_{i}$ and $\sum_{j=1}^{d_{i}} z_{i}\left(z_{i}^{j}\right)=1$, where $d_{i}=\operatorname{card}\left(Z_{i}\right)$.

(2) A pure strategy $z_{i}$ is a degenerate mixed strategy that assigns probability 1 to $z_{i}$ and probability 0 to all other pure strategies of player $i$.

(3) For any player $i \in I$, and any mixed strategy $z_{i} \in \mathcal{Z}_{i}$, we define the support of $z_{i}$, denoted $\operatorname{supp}\left(z_{i}\right)$, by $\operatorname{supp}\left(z_{i}\right)=\left\{z_{i}^{j} \in Z_{i} ; z_{i}\left(z_{i}^{j}\right)>0\right\}$. It means that the support of $z_{i}$ is the set of pure strategies $z_{i}^{j}$ for which correspond strictly positive components in vector $z_{i}$.

Definition 5.1. A mixed strategy profile $z^{\star} \in Z$ is a mixed unilateral support equilibrium of game $\Gamma_{n}$ if,

$$
\forall i \in I, \forall j \in I \backslash\{i\}, p_{i}\left(z_{-j}^{\star}, z_{j}^{\star}\right) \geq p_{i}\left(z_{-j}^{\star}, z_{j}\right) ; \forall z_{j} \in \mathcal{Z}_{j} .
$$

Definition 5.2. The set of Best Individualistic Support to player $i$, offered by player $j$, given a fixed incomplete profile of mixed strategies $z_{-j}$ is defined by:

$$
\operatorname{BIS}_{i, j}\left(z_{-j}\right)=\left\{z_{j} \in \mathcal{Z}_{j} ; p_{i}\left(z_{-j}, z_{j}\right) \geq p_{i}\left(z_{-j}, z_{j}^{\prime}\right), \forall z_{j}^{\prime} \in \mathcal{Z}_{j}\right\}
$$

Using this set, we write Definition 5.1 as:

Theorem 5.3. A profile of mixed strategies $z^{\star}=\left(z_{-i}^{\star}, z_{i}^{\star}\right)$ is a mixed profile USE of $\Gamma_{n}$ if, and only if, $\forall i \in I$, $\forall j \in I \backslash\{i\}$, we have $z_{j}^{\star} \in \operatorname{BIS}_{i, j}\left(z_{-j}^{\star}\right)$.

Proof. The proof of this theorem is evident from the definition of the set $\mathrm{BIS}_{i, j}$.

Lemma 5.4. Let $\Gamma_{n}$ be a finite strategic game. Then, $z^{\star}$ is a mixed profile USE of $\Gamma_{n}$ if, and only if, for every player $i \in I$, for every player $j \in I \backslash\{i\}$, every pure strategy in the support of $z_{j}^{\star}$ is a Best Individualistic Support to player $i$, offered by player $j$, given a fixed incomplete profile of mixed strategies $z_{-j}^{\star}$. 
Proof. We use in this proof the "reductio ad absurdum" method. For that, we consider $z^{\star}$ a mixed profile USE of $\Gamma_{n}$ and we suppose that $\exists$ a player $i, \exists$ a player $j \neq i$ such that $z^{\star}=\left(z_{-i}^{\star}, z_{i}^{\star}\right)=\left(z_{1}, \ldots, z_{j}^{\star}, \ldots, z_{i}^{\star} \ldots, z_{n}^{\star}\right)$, for which $\exists z_{j} \in \operatorname{supp}\left(z_{j}^{\star}\right)$ a pure strategy such that $z_{j} \notin \operatorname{BIS}_{i, j}\left(z_{-j}^{\star}\right)$. Then, by linearity and by the expansion of $p_{i}$, player $j$ will seek for another pure strategy, denoted by $z_{j}^{\prime}$ where $p_{i}\left(z_{-j}^{\star}, z_{j}^{\prime}\right)>p_{i}\left(z_{-j}^{\star}, z_{j}\right)$, this $z_{j}^{\prime}$ exists since $z^{\star}$ is a mixed USE. Therefore, if we expand $p_{i}\left(z_{-j}^{\star}, z_{j}^{\star}\right)$ which depends on the term $z_{j}^{\star}\left(z_{j}\right) p_{i}\left(z_{-j}^{\star}, z_{j}\right)+z_{j}^{\star}\left(z_{j}^{\prime}\right) p_{i}\left(z_{-j}^{\star}, z_{j}^{\prime}\right)$, the player $j$ has to increase rationally the probability of playing the pure strategy $z_{j}^{\prime}$ instead of $z_{j}$ since all players have individualistic support behavior. Thus, by transfer of probability, player $i$ can increase the coefficient of his payoff $p_{i}\left(z_{j}^{\prime}, z_{-j}^{\star}\right)$ by adding $z_{j}^{\star}\left(z_{j}\right)$ to $z_{j}^{\star}\left(z_{j}^{\prime}\right)$ and obtaining the term $0+\left(z_{j}^{\star}\left(z_{j}\right)+z_{j}^{\star}\left(z_{j}^{\prime}\right)\right) p_{i}\left(z_{-j}^{\star}, z_{j}^{\prime}\right)$ holding a payoff greater than the old term $\left[z_{j}^{\star}\left(z_{j}\right) p_{i}\left(z_{-j}^{\star}, z_{j}\right)+z_{j}^{\star}\left(z_{j}^{\prime}\right) p_{i}\left(z_{-j}^{\star}, z_{j}^{\prime}\right)\right]$. Let $z_{j}^{\text {Transfer }}=\left(z_{j}^{\star}\left(z_{j}\right)+z_{j}^{\star}\left(z_{j}^{\prime}\right)\right)$. Then, $p_{i}\left(z_{-j}^{\star}, z_{j}^{\text {Transfer }}\right)>p_{i}\left(z_{-j}^{\star}, z_{j}^{\star}\right)$. It yields that $z_{j}^{\star} \notin \operatorname{BIS}_{i, j}\left(z_{-j}^{\star}\right)$ and then $z^{\star}$ is not a mixed USE. Contradiction.

We continue by proving the second implication. We will use here the contrapositive method. We start by supposing that " $z^{\star}=\left(z_{-i}^{\star}, z_{i}^{\star}\right)=\left(z_{1}, \ldots, z_{j}^{\star}, \ldots, z_{i}^{\star} \ldots, z_{n}^{\star}\right)$ is not a mixed profile USE of $\Gamma_{n}$ ". It means that $\exists i$ and $\exists j \neq i$ such that $z_{j}^{\star} \notin \operatorname{BIS}_{i, j}\left(z_{j}^{\star}\right)$. Thus, $\exists$ a mixed strategy $z_{j}^{\prime} \in \operatorname{BIS}_{i, j}\left(z_{j}^{\star}\right)$, where $p_{i}\left(z_{-j}^{\star}, z_{j}^{\prime}\right)>$ $p_{i}\left(z_{-j}^{\star}, z_{j}^{\star}\right)$. Therefore, we can conclude that at least one pure strategy $z_{j} \in \operatorname{supp}\left(z_{j}^{\star}\right)$ holds a payoff less than an other pure strategy $z_{j}^{\prime} \in \operatorname{supp}\left(z_{j}^{\prime}\right)$ i.e. $p_{i}\left(z_{-j}, z_{j}\right)<p_{i}\left(z_{-j}, z_{j}^{\prime}\right)$ since $p_{i}$ is linear (see [28]). Consequently, $z_{j} \notin \operatorname{BIS}_{i, j}\left(z_{j}^{\star}\right)$.

This lemma provides us with an efficient method used to calculate probability distributions relative to mixed USE. The lemma result is as follows: for each player $i$, every strategy in the support of an equilibrium mixed strategy of each player $j \neq i$, individually, yields that player $i$ the same payoff.

Example: We consider the game $\Gamma_{3}=\left\langle\{1,2,3\},\left(Z_{i}\right)_{i=1}^{3},\left(\mathcal{Z}_{i}\right)_{i=1}^{3},\left(p_{i}\right)_{i=1}^{3}\right\rangle$, where $Z_{i}$ is a finite set; $\forall i=1,2,3$.

- We consider that the pure strategies of player $i$ are $: z_{i}^{1}$ and $z_{i}^{2}, \forall i=1,2,3$.

- The mixed strategies of player $i$ are $: z_{i}^{1}$ and $z_{i}^{2}, \forall i=1,2,3$.

The only case that will be developed in the following and in example 5.1.1, is under the assumption that the mixed unilateral support equilibrium support is equal to $Z_{i}$, for all $i$. Then, according to Lemma 5.4, we find the mixed unilateral support equilibrium by writing the following system:

- $p_{1}\left(z_{1}, z_{2}^{1}, z_{3}\right)=p_{1}\left(z_{1}, z_{2}^{2}, z_{3}\right)$ and $p_{1}\left(z_{1}, z_{2}, z_{3}^{1}\right)=p_{1}\left(z_{1}, z_{2}, z_{3}^{2}\right)$.

- $p_{2}\left(z_{1}^{1}, z_{2}, z_{3}\right)=p_{2}\left(z_{1}^{2}, z_{2}, z_{3}\right)$ and $p_{2}\left(z_{1}, z_{2}, z_{3}^{1}\right)=p_{2}\left(z_{1}, z_{2}, z_{3}^{2}\right)$.

- $p_{3}\left(z_{1}^{1}, z_{2}, z_{3}\right)=p_{3}\left(z_{1}^{2}, z_{2}, z_{3}\right)$ and $p_{3}\left(z_{1}, z_{2}^{1}, z_{3}\right)=p_{3}\left(z_{1}, z_{2}^{2}, z_{3}\right)$.

\subsection{Tensor representation of mixed Unilateral Support Equilibrium}

We proved a theorem dedicated to calculate mixed USE profiles. Similar theorems existed for Nash mixed equilibrium in [28] and for Berge mixed equilibrium in [29]. However, the classic formula to calculate expected payoffs in game theory seems complicated when dealing with more than 3 players in a game. The method to deal with these types of games and to calculate the expected payoffs, more easily, is presented in the game's tensor form. Especially, the Mode $-i$ vector product manages the multiplication between tensors and vectors and allows dealing with high dimensional USE equilibrium problems. We recall the definition of this operation, which can be found in $[18,20]$, as follows:

Definition 5.5. The Mode $-i$ (vector) product of a tensor $T=\left(T_{i_{1} i_{2} \ldots i_{n}}\right) \in \mathbb{R}^{d_{1} \times d_{2} \times \ldots \times d_{i} \times \ldots \times d_{n}}$ with a vector $V \in \mathbb{R}^{d_{i}}$ is denoted by $U=T \overline{\times}_{i} V$, which is a real $(n-1)$ th order $d_{1} \times d_{2} \times \ldots \times d_{i-1} \times d_{i+1} \times \ldots \times d_{n}$-dimensional tensor with

$$
U_{i_{1} \times i_{2} \times \ldots \times i_{i-1} \times i_{i+1} \times \ldots \times i_{n}}=\sum_{i_{k}=1}^{d_{k}} T_{i_{1} i_{2} \ldots i_{n}} V_{i_{k}}
$$

for any $i_{j} \in\left\{1,2, \ldots, d_{j}\right\}$ with $j \in\{1,2, \ldots, n\} \backslash\{k\}$. 
This tensor tool gives tensor form the legitimacy to deal with all kind of high dimensional equilibrium problems, and then strengthen the reason to use tensor form for $n$ greater than 3 players. We start by exploiting the method to calculate expected payoffs using tensors and then to find USE mixed equilibrium in a 7-player game under the result of Lemma 5.4. Let $\Gamma_{n}=\left\langle I,\left(Z_{i}\right)_{i \in I},\left(\mathcal{Z}_{i}\right)_{i \in I},\left(p_{i}\right)_{i \in I}\right\rangle$ be a game. A tensor form of $\Gamma_{n}$ is given by $n$ tensors $T^{(i)}$, where $i \in\{1, \ldots, n\}$, respectively for each one of the $n$ players, and merged in a multi-tensor $\mathcal{T}$. Then, the expected payoff function $p_{i}(z)$ for $z \in \mathcal{Z}=\underset{i=1}{n} \mathcal{Z}_{i}$ is defined as follows:

$$
p_{i}(z)=T^{(i)} \overline{\times}_{n} z_{n} \overline{\times}_{n-1} z_{n-1} \overline{\times}_{n-2} \ldots \overline{\times}_{2} z_{2} \overline{\times}_{1} z_{1} \quad \text { for } i=1,2, \ldots, n .
$$

Note that starting by Mode $-i$ vector product for $i=n$ and continuing in a descending order until $i=1$ is necessary because it assures a well-posed multiplication between tensors and Mode $-k$ fibers for $k=1,2, \ldots, n$. Now, after defining the expected payoffs using tensors, we use it in a numerical example to clarify the consequence of Lemma 5.4.

\subsubsection{Numerical example: a 7-player game}

We consider a game $\Gamma_{7}=\left\langle I=\{1,2,3,4,5,6,7\},\left(Z_{1}, Z_{2}, Z_{3}, Z_{4}, Z_{5}, Z_{6}, Z_{7}\right),\left(\mathcal{Z}_{1}, \mathcal{Z}_{2}, \mathcal{Z}_{3}, \mathcal{Z}_{4}, \mathcal{Z}_{5}, \mathcal{Z}_{6}, \mathcal{Z}_{7}\right)\right.$, $\left.\left(p_{1}, p_{2}, p_{3}, p_{4}, p_{5}, p_{6}, p_{7}\right)\right\rangle$, where $Z_{i}=\left\{z_{i}^{1}, z_{i}^{2}, z_{i}^{3}\right\}$. We recall that $z_{i}=\left(z_{i}^{1}, z_{i}^{2}, \ldots, z_{i}^{7}\right)$ is a probability distribution. In addition, the tensor form of $\Gamma_{7}$ is given by seven tensors $T^{(i)} \in \mathbb{R}^{3 \times 3 \times 3 \times 3 \times 3 \times 3 \times 3}$, where $T^{(i)}=p_{i}\left(z_{1}, z_{2}, z_{3}, z_{4}, z_{5}, z_{6}, z_{7}\right)$ for $i \in\{1,2,3,4,5,6,7\}$. These tensors can be merged in a multi-tensor $\mathcal{T} \in \mathbb{R}^{3 \times 3 \times 3 \times 3 \times 3 \times 3 \times 3}$. $\mathcal{T}$ contains then $7 \times 3^{7}$ payoffs. Each payoff is given by the counter $k$ varying from 1 to the last node $\left(7 \times 3^{7}\right)$ in the seventh tensor, using the formula $k \equiv p_{i}\left(z_{1}, z_{2}, z_{3}, z_{4}, z_{5}, z_{6}, z_{7}\right)(\bmod 2)+1$. As a consequence of Lemma 5.4, USE mixed profiles of $\Gamma_{7}$, when they exist, are solutions to the following system:

$$
\begin{aligned}
\forall i \in I, \quad & p_{i}\left(z_{1}, \ldots, z_{j}^{1}, \ldots, z_{i}, \ldots, z_{7}\right)= \\
& p_{i}\left(z_{1}, \ldots, z_{j}^{2}, \ldots, z_{i}, \ldots, z_{7}\right)= \\
& p_{i}\left(z_{1}, \ldots, z_{j}^{3}, \ldots, z_{i}, \ldots, z_{7}\right), \quad \forall j \in I \backslash\{i\} .
\end{aligned}
$$

This system (5.3) must hold for all players $i$. In other words, in each member of the equation, a player $j \neq i$ holds one of his three pure strategies, and that for all $j \neq i$, when all other players keep playing their fixed mixed strategies. However, because expected payoffs are difficult to expand using the classical way as in $[16,17]$, tensors used with system (5.3) addresses equation (5.2) in a systematic way as follows:

$$
\begin{aligned}
\forall i \in I, \quad & \left(\left(\left(\left(\left(T^{(i)} \overline{\times}_{7} z_{7}\right) \ldots \overline{\times}_{i} z_{i}\right) \ldots \overline{\times}_{j} z_{j}^{1}\right) \ldots \overline{\times}_{1} z_{1}\right)\right)= \\
& \left(\left(\left(\left(\left(T^{(i)} \overline{\times}_{7} z_{7}\right) \ldots \overline{\times}_{i} z_{i}\right) \ldots \overline{\times}_{j} z_{j}^{2}\right) \ldots \overline{\times}_{1} z_{1}\right)\right)= \\
& \left(\left(\left(\left(\left(T^{(i)} \overline{\times}_{7} z_{7}\right) \ldots \overline{\times}_{i} z_{i}\right) \ldots \overline{\times}_{j} z_{j}^{3}\right) \ldots \overline{\times}_{1} z_{1}\right)\right), \forall j \in I \backslash i
\end{aligned}
$$

We can expand this system using tensor class on Matlab, see [4], and using "symbolic" variables. We obtain a system of 126 equations to be solved, with 21 unknowns. Using the fact that $z_{i}$ is a probability distribution, for each $i$, the number of unknowns decreases then to 14 by involving in the system the equations $z_{i}^{3}=1-z_{i}^{2}-z_{i}^{1}, \forall i \in$ $I=\{1,2, \ldots, 7\}^{2}$. The resulted non-linear system (5.4) is solved using the algorithm "trust-region-reflective",

\footnotetext{
${ }^{2}$ The equations of this system can be provided upon the readers' request.
} 
see [22]. The solution of this system reveals the following mixed unilateral support equilibrium:

$$
\begin{aligned}
& z_{1}=(0.5,0.5,0) \\
& z_{2}=(0.5,0.5,0) \\
& z_{3}=(0.5,0.5,0) \\
& z_{4}=(0.5,0.5,0) \\
& z_{5}=(0.5,0.5,0) \\
& z_{6}=(0.5,0.5,0) \\
& z_{7}=(0.5,0.5,0) .
\end{aligned}
$$

\section{Conclusion}

In this paper, we have localized pure unilateral support equilibrium within pure Nash and pure Berge equilibria by introducing the tensor form. We have also implemented an algorithm based on tensors as main variables to solve USE high dimensional problems. Our definition of a new set called Best Individualistic Support, dedicated to unilateral support equilibrium, provides an extension of pure USE to mixed USE, when the set of pure strategies of each player is finite. We have also proposed and proved a lemma yielding a method to calculate mixed unilateral support equilibrium profiles. Our results complete those on math computation of pure USE, prepare the field of mixed USE, and introduce to the literature an efficient method based on tensors for USE future research.

For further research, a new equilibrium seems to be well defined as a consequence of tensor form structure applied to Nash, Berge and Unilateral Support equilibria. We showed in Nash equilibrium tensor form that the procedure is based on Mode $-i$ fiber for each player $i$, see the interpretation of (3.2). Moreover, USE tensor procedure is based on all Mode - $j$ fibers without Mode $-i$ fiber (for $j \neq i$ ), see the interpretation of (3.6). However, the tensor procedure to compute Berge equilibrium is based on all subarrays depending on player $i$, see the interpretation of (3.4). Then, a new equilibrium tensor procedure would be based on all subarrays depending on the group of players- $j$ without player $j$. As we showed for USE that each player $i$ is supported by a player $j$ individually for each $j \neq i$, then the new equilibrium can have an interpretation as follows: for each player $i$, and for each player $j \neq i$, player $i$ is supported by the group of players- $j$.

Acknowledgements. The authors would like to especially thank professor Tarik Tazdaït for his very valuable assistance. Both authors are very grateful to the editor and the two referees for their valuable suggestions and remarks which considerably improved this paper. The authors' work was supported by the research project "PHC CEDRE 2019" No: 42315RA.

\section{REFERENCES}

[1] K. Abalo and M. Kostreva, Some existence theorems of nash and berge equilibria. Appl. Math. Lett. 17 (2004) $569-573$.

[2] K. Abalo and M. Kostreva, Berge equilibrium: some recent results from fixed-point theorems. Appl. Math. Comput. 169 (2005) 624-638.

[3] J.E. Abdou, E. Safatly, B. Nakhle and A. El Khoury, High-dimensional nash equilibria problems and tensors applications. Int. Game Theory Rev. 19 (2017) 1750015.

[4] B.W. Bader, T.G. Kolda, et al. Matlab tensor toolbox version 2.6. Available online (2015).

[5] C. Berge, Théorie générale des jeux à n personnes. In Vol. 138 of Mémorial des sciences mathématiques. Gauthier-Villars (1957).

[6] R. Bishop and S. Goldberg, Tensor analysis on manifolds. Dover Books on Mathematics. Dover Publications (1968).

[7] B.R. Cobb and T. Sen, Finding mixed strategy nash equilibria with decision trees. Int. Rev. Econ. Edu. 15 (2014) 43-50.

[8] A.M. Colman, T.W. Körner, O. Musy and T. Tazdaït, Mutual support in games: some properties of berge equilibria. J. Math. Psychol. 55 (2011) 166-175.

[9] H.W. Corley, A mixed cooperative dual to the nash equilibrium. Game Theory 2015 (2015) 7.

[10] P. Courtois, R. Nessah and T. Tazdaït, How to play games? Nash versus berge behaviour rules. Econ. Philos. 31 (2015) 123-139. 
[11] P. Courtois, R. Nessah and T. Tazdaït, Existence and computation of berge equilibrium and of two refinements. J. Math. Econ. 72 (2017) 7-15.

[12] B. Crettez, A new sufficient condition for a berge equilibrium to be a Berge-Vaisman equilibrium. J. Quant. Econ. 15 (2017) 451-459.

[13] B. Crettez, On sugden's "mutually beneficial practice" and berge equilibrium. Int. Rev. Econ. 64 (2017) $357-366$.

[14] B. Crettez, Unilateral support equilibrium, berge equilibrium, and team problems solutions. J. Quant. Econ. 17 (2019) $727-739$.

[15] B. Crettez and R. Nessah, On the existence of unilateral support equilibrium. Math. Soc. Sci. 105 (2020) 41-47.

[16] D. Fudenberg and J. Tirole, Game Theory. MIT Press, Cambridge, MA, USA (1991).

[17] R. Gibbons, Game Theory for Applied Economists. Princeton University Press, Princeton, NJ, USA (1992).

[18] Z.-H. Huang and L. Qi, Formulating an n-person noncooperative game as a tensor complementarity problem. Comput. Optim. Appl. 66 (2017) 557-576.

[19] H.A.L. Kiers, Towards a standardized notation and terminology in multiway analysis. J. Chemom. 14 (2000) $105-122$.

[20] T.G. Kolda and B.W. Bader, Tensor decompositions and applications. SIAM Rev. 51 (2009) 455-500.

[21] M. Larbani and R. Nessah, A note on the existence of berge and berge-nash equilibria. Math. Soc. Sci. 55 (2008) $258-271$.

[22] Y. Li, Centering, trust region, reflective techniques for nonlinear minimization subject to bounds. Technical report, Ithaca, NY, USA (1993).

[23] O. Musy, A. Pottier and T. Tazdaït, A new theorem to find berge equilibria. Int. Game Theory Rev. 14 (2012) 1250005.

[24] J.F. Nash, Equilibrium points in n-person games. Proc. Nat. Acad. Sci. 36 (1950) 48-49.

[25] R. Nessah and M. Larbani, Berge-Zhukovskii equilibria: existence and characterization. Int. Game Theory Rev. 16 (2014) 1450012.

[26] R. Nessah, M. Larbani and T. Tazdaït, A note on berge equilibrium. Appl. Math. Lett. 20 (2007) 926-932.

[27] J.V. Neumann and O. Morgenstern, Theory of Games and Economic Behavior. Princeton University Press, Princeton, NJ, USA (1944).

[28] M.J. Osborne and A. Rubinstein, A Course in Game Theory. MIT Press, Cambridge, MA, USA (1994).

[29] E. Safatly and J.E. Abdou, Locating pure and mixed berge equilibria using tensor form. Submitted (2019).

[30] J. Schouten, P. Borm and R. Hendrickx, Unilateral support equilibria. J. Math. Psychol. 93 (2019) 102295.

[31] C. Semay and B. Silvestre-Brac, Introduction au calcul tensoriel: Applications à la physique. Sciences sup. Dunod (2007).

[32] R.L. Trivers, The evolution of reciprocal altruism. Q. Rev. Biol. 46 (1971) 35-57.

[33] V.I. Zhukovskii, Some problems of nonantagonistic differential games. In Matematiceskie Metody v Issledovanii Operacij [Mathematical Methods in Operations Research] Edited by P. Kenderov. Bulgarian Academy of Sciences (1985) 103-195. 